\title{
Submm/FIR CO line emission from the disk of the Class I protostar EL 29^
}

\author{
C. Ceccarelli ${ }^{1,2}$, A. C. A. Boogert ${ }^{3,4}$, A. G. G. M. Tielens ${ }^{3}$, E. Caux ${ }^{5}$, M. R. Hogerheijde ${ }^{6}$, and B. Parise ${ }^{5}$ \\ 1 Observatoire de Bordeaux, BP 89, 33270 Floirac, France \\ ${ }^{2}$ Laboratoire d'Astrophysique, Observatoire de Grenoble, BP 53, 38041 Grenoble Cedex 09, France \\ 3 California Institute of Technology, Department of Astronomy 105-25, Pasadena, CA 91125, USA \\ 4 Kapteyn Astronomical Insitute, PO Box 800, 9700 AV Groningen, The Netherlands \\ 5 CESR CNRS-UPS, BP 4346, 31028, Toulouse cedex 04, France \\ ${ }^{6}$ Steward Observatory, The University of Arizona, 933 N. Cherry Ave - Tucson, AZ 85721-0065, USA
}

Received 26 April 2002 / Accepted 27 August 2002

\begin{abstract}
We present observations towards the Class I protostar EL 29 of the CO $J=6 \rightarrow 5$ and $J \geq 15$ transitions obtained with JCMT and ISO LWS respectively, and of five $\mathrm{H}_{2}$ rotational lines obtained with ISO SWS. The simultaneous analysis of these observations reveals the presence of a warm gas component at about 170-250 K, $\sim 550 \mathrm{AU}$ in size, and whose density is $\geq 10^{6} \mathrm{~cm}^{-3}$. The mass of the warm gas is $\sim 8-24 \times 10^{-4} M_{\odot}$. These values compare extremely well with the predictions of the temperature and mass of the flaring disk surrounding EL 29, probed by the dust continuum. We propose that the observed FIR $\mathrm{CO}$ emission originates in the super-heated surface layer of the disk of EL 29 and discuss its characteristics. We find that the $\mathrm{CO}$ abundance in the disk is large, $\geq 10^{-4}$, implying no depletion or photodissociation and we present arguments in favor of the idea that the dust has settled on the midplane disk of EL 29 and that it is thermally decoupled from the gas. We compare the characteristics of the El 29 disk with those of the disks observed in other Herbig AeBe stars using recent studies of $\mathrm{H}_{2}$ rotational line emission. The gas temperature and mass derived for the disk of EL 29 are similar to the disks of the previously studied sample. In EL 29, as in Herbig AeBe stars, the gas and dust are probably thermally decoupled. Finally, the upper limit on the $\mathrm{H}_{2} \mathrm{O}$ emission that we obtain suggests that water is not an important coolant of the disk gas, in agreement with theoretical water abundance predictions. The present study challenges previous claims that the FIR CO line emission observed in sources similar to EL 29 originates in shocks.
\end{abstract}

Key words. stars: formation - ISM: lines and bands - ISM: individual objects: EL 29 - stars: circumstellar matter

\section{Introduction}

Far InfraRed (FIR) CO emission, lines with $J \geq 14$, has been observed by ISO in several low mass protostars, both Class 0 or Class I objects, to which they belong (a recent review is given in Ceccarelli 2000). This FIR CO emission usually has been attributed to the shocked gas at the interface between the outflowing gas and the surroundings (e.g. Giannini et al. 2001 and references therein). The reason for that is the relatively high temperature, $\geq 200 \mathrm{~K}$ on a scale of at least a few hundred AUs, derived by the analysis of the $\mathrm{CO}$ spectra, which has been taken as a sign of extra heating, other than the radiation from the central source. However, the shock interpretation has some difficulties. First, if the FIR CO emission originates in shocked gas, water emission is expected to

Send offprint requests to: C. Ceccarelli,

e-mail: Cecilia.Ceccarelli@observ.u-bordeaux.fr

^ Based on observations with ISO, an ESA project with instruments funded by ESA Member States (especially the PI countries: France, Germany, the Netherlands and the United Kingdom) with the participation of ISAS and NASA. be bright as well, as water is expected to be copiously formed under such conditions. The expectations are based on theoretical modeling (e.g. Hollenbach \& McKee 1989; Kaufman \& Neufeld 1996) and on the actual observation of abundant water in clear-cut shocked regions, like HH54B (Liseau et al. 1996) and Orion (Harwit et al. 1998; Cernicharo et al. 1999). On the contrary, water emission is only observed towards Class 0 sources and it seems to be more correlated with the mass of the envelope surrounding those sources than with their $\mathrm{SiO}$ emission - usually considered a shock tracer of (Ceccarelli et al. 1999). Indeed, the water emission observed towards the two Class 0 sources IRAS16293-2422 and NGC 1333-IRAS4 has been successfully explained as due to the thermal emission from their envelopes ${ }^{1}$ (Ceccarelli et al. 2000; Maret et al. 2002). In addition, water emission is usually stronger towards the central positions and weaker, when detected, towards the emission peaks of millimeter $\mathrm{CO}$ and

\footnotetext{
${ }^{1}$ IRAS16293-2422 and NGC 1333-IRAS4 are the only sources where an accurate modeling of water emission from the envelope has been carried out so far.
} 
other shock tracers, like SiO for example (Caselli et al. 1997; Schilke et al. 1997). A notable example is L1448 (Nisini et al. 2000), where the strongest water emission is detected at the two ISO positions encompassing the two Class 0 sources of the region, L1448-mm and L1448-N, rather than at the outflow south lobe, which shows bright millimeter emission from a large variety of molecules (Bachiller et al. 1990; Curiel et al. 1999). Another example is IRAS16293-2422, where very bright water emission is detected only towards the central position and is undetected towards the bright $\mathrm{SiO}$ and $\mathrm{H}_{2} \mathrm{CO}$ (Castets et al. 2001), methanol (Garay et al. 2002), and SO/SO (Wakelam et al. 2002) emission peaks. Other examples include NGC 1333-IRAS4 (Maret et al. 2002) and IRAS2 (Caux et al. 1999). In summary, if the observed FIR CO emission originates in shocks close to the central object, those shocks do not seem to produce much water, which is a somewhat troubling result requiring additional explanation. Explanation of the water under-abundance is certainly possible and includes depletion of oxygen and/or water into grain mantles, as well as re-condensation on grains (e.g. Bergin et al. 1999).

In this paper we will not discuss directly the "water problem", but we challenge the thesis that the FIR CO emission is emitted by shocked gas. For this we analyze the case of EL 29, a well-studied $36 L_{\odot}$ (distance 160 pc; Chen et al. 1995) Class I source in the $\rho$ Ophiuchus complex (Wilking et al. 1989), on which we carried out an extensive observational study of the millimeter to near infrared emission (Boogert et al. $2000,2002)$. In this paper we present (Sect. 2) ISO observations in the 43-197 $\mu \mathrm{m}$ spectral range obtained with the Long Wavelength Spectrometer (LWS; Clegg et al. 1996), observations of the $\mathrm{H}_{2}$ rotational transitions obtained with the Short Wavelength Spectrometer (SWS; de Graauw et al. 1996), and a map of the $\mathrm{CO} J=6 \rightarrow 5$ emission obtained with the James Clerk Maxwell Telescope (JCMT). The goal is to pin down the origin of the FIR CO emission combining the FIR and the $J=6 \rightarrow 5$ observations, and the further constraint provided by the $\mathrm{H}_{2}$ observations. The two sets of $\mathrm{CO}$ line observations are complementary, as LWS ISO observations have a relatively poor spatial (beam $F W H M \Delta \theta \sim 80^{\prime \prime}$ ) and spectral $\left(\Delta v \sim 1500 \mathrm{~km} \mathrm{~s}^{-1}\right)$ resolution, but probe warm and dense gas, whereas $J=6 \rightarrow 5$ observations provide much better access to the spatial extent $\left(\Delta \theta \sim 12^{\prime \prime}\right)$ and kinematics $\left(\Delta v \sim 0.6 \mathrm{~km} \mathrm{~s}^{-1}\right)$ of the warm gas. Finally, $\mathrm{H}_{2}$ observations are a crucial test for the gas column density in the line of sight.

Using all this information we are able to constrain very accurately the temperature and mass of the emitting gas (Sect. 4), and, consequently, to discuss the origin of the observed CO FIR emission, which we propose is the flaring disk of EL 29 (Sect. 5).

\section{Observations}

a) $C O J=6 \rightarrow 5$ observations

The CO $J=6 \rightarrow 5$ line $(691.473 \mathrm{GHz})$ was observed with the "RxG" receiver on JCMT (Mauna Kea, Hawaii USA) in April 1995. Technical details on the performance of this receiver at the JCMT can be found in Harris et al. (1994). The spectral resolution was $0.61 \mathrm{~km} \mathrm{~s}^{-1}$ per channel.
The JCMT's beam is described by a composite beam containing $55 \%$ of the power in a $7 "$ FWHM Gaussian, and $45 \%$ in an $18^{\prime \prime} F W H M$ Gaussian, giving an effective beam equal to $12^{\prime \prime}$. The data has been converted from a $T_{\mathrm{A}}^{*}$ to $T_{\mathrm{R}}^{*}$ scale, applying an efficiency factor $\eta_{\mathrm{fss}}=0.27$, which corrects for all instrumental scattering and loss terms. The weather conditions were excellent, with a transmission at zenith of 0.6 at the CO $J=6 \rightarrow 5$ frequency. We obtained a $5 \times 5$ point map spaced by 7 ", covering a $35^{\prime \prime} \times 35^{\prime \prime}$ field centered on $\operatorname{RA}(1950.0)=16^{\mathrm{h}} 24^{\mathrm{m}} 07^{\mathrm{s}} .7, \operatorname{Dec}(1950.0)=-24^{\mathrm{o}} 30^{\prime} 37^{\prime \prime}$. The effective integration time on each position was 1 minute, yielding a rms noise level of $T_{\mathrm{R}}^{*}=1.9 \mathrm{~K}$. Additionally, we observed the ${ }^{13} \mathrm{CO} J=6 \rightarrow 5$ line (at $661.067 \mathrm{GHz}$ ) towards the central position and towards $\triangle \mathrm{RA}, \triangle \mathrm{DEC}=-14^{\prime \prime},+14^{\prime \prime}$, i.e. at the edge of the ${ }^{12} \mathrm{CO}$ map. The effective integration time was $4 \mathrm{~min}$ yielding rms noise levels of $1.0 \mathrm{~K}$. The observations were done in the beam switching mode, with the chop throw alternated between $+120^{\prime \prime}$ (reference position A) and $-120^{\prime \prime}$ (position B) in Azimuth. Contamination by CO $J=6 \rightarrow 5$ emission in the A or $\mathrm{B}$ reference positions was checked by subtracting the spectra obtained with the A and B reference positions respectively. Both the averaged and subtracted spectra are given in Fig. 1.

\section{b) ISO observations}

EL 29 was observed during Revolution 484 (14th March 1997) with the ISO-LWS. We obtained 15 scans covering the range from $43 \mu \mathrm{m}$ to $197 \mu \mathrm{m}$ in the low resolution mode $(R \sim 200)$ for a total of $2611 \mathrm{~s}$ of integration time. We also obtained other 15 scans toward an off-source position, at RA(2000) $16^{\mathrm{h}} 27^{\mathrm{m}} 09.3^{\mathrm{s}}$ and $\operatorname{DEC}(2000)-24^{\circ} 35^{\prime} 18.1^{\prime \prime}$. The data were reduced using the Off-Line-Processing (OLP) version 10 and the ISO-Spectral-Analysis-Package (ISAP) version 2.1. The spectra were flux calibrated using Uranus (Swinyard et al. 1996) and the absolute accuracy is estimated to be better than $30 \%$. Finally, the LWS beamsize is roughly constant at all wavelengths, namely $\sim 80^{\prime \prime}$ (Swinyard et al. 1998). Note that these observations measure absolute fluxes, i.e. they are not "beam-switched", as is the case for the JCMT observations reported in the previous paragraph.

We searched for five pure rotational lines of molecular hydrogen by using ISO-SWS: S(1), S(2), S(4), S(5) and $S(6)$. The observations were performed during revolution 267 (August 1996) and 292 (September 1996) in the SW1 and SW6 modes respectively, with a spectral resolution of $\lambda / \Delta \lambda$ equal to 400 and 1500 respectively. The SWS aperture is equal to $20^{\prime \prime} \times 27^{\prime \prime}$ and $14^{\prime \prime} \times 27^{\prime \prime}$ at the $\mathrm{S}(0)$ and $\mathrm{S}(1)$ transitions respectively, and $14^{\prime \prime} \times 20^{\prime \prime}$ at the other ones. The absolute flux calibration is accurate to $20 \%$. More details on the data reduction are reported in Boogert et al. (2000).

\section{Results}

\section{1. $\mathrm{CO} J=6 \rightarrow 5$ emission}

$\mathrm{CO} J=6 \rightarrow 5$ emission is elongated in the NW-SE direction. Detailed information about the morphology of the region can be found in Boogert et al. (2002; hereinafter BHC02), who report single dish and interferometric observations of several molecular transitions. Here we give a short summary to help 


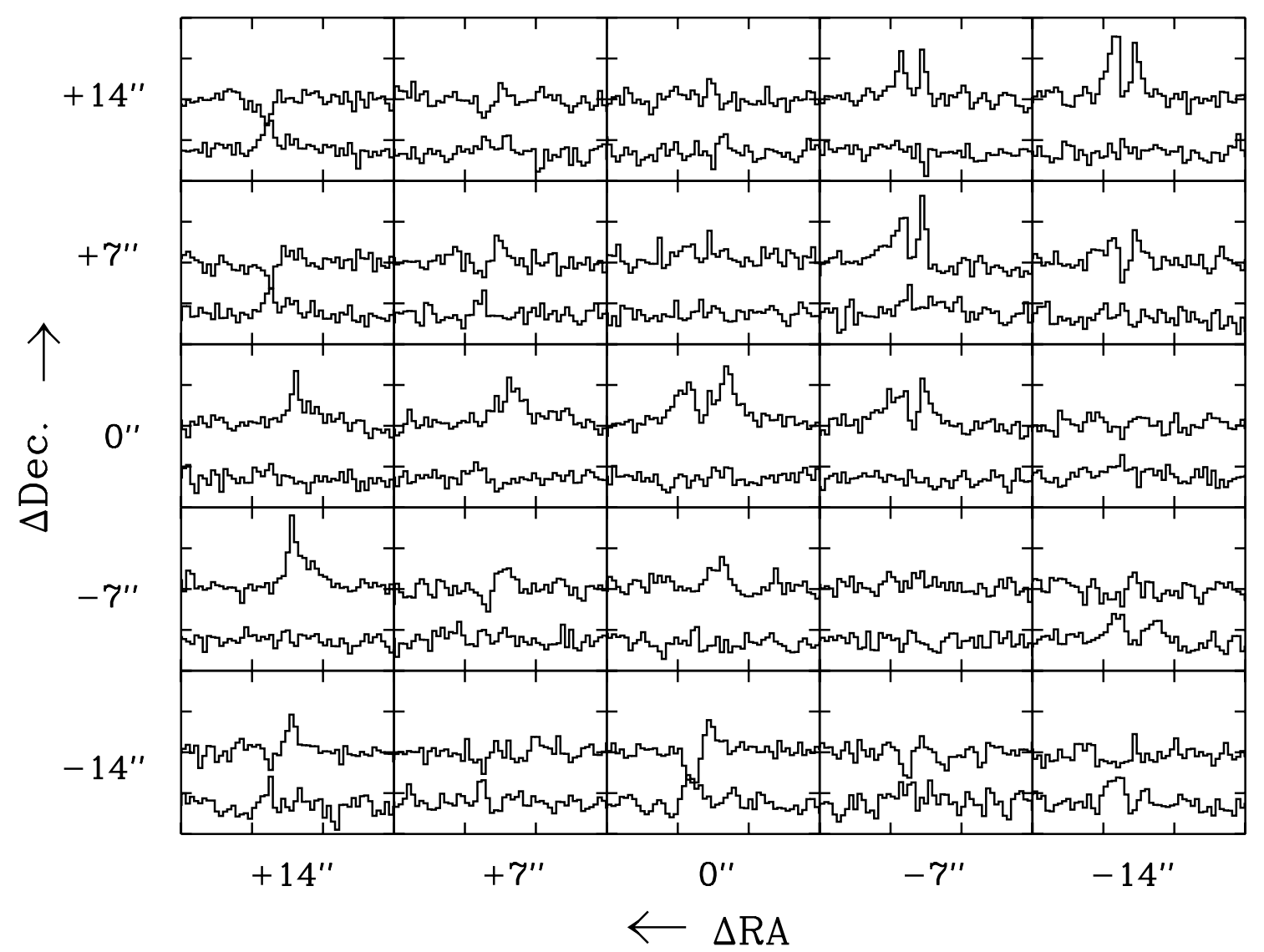

Fig. 1. Map of the ${ }^{12} \mathrm{CO} J=6 \rightarrow 5$ emission. The map is centered on the infrared position (see text). Each panel gives the average of the spectra obtained with respect to the A and B sky background positions, i.e. "(A+B)/2" (top), as well as the difference between these A and B spectra, "(A-B)/2", which was shifted by $-20 \mathrm{~K}$ for clarity (bottom). Any signal in the difference spectrum is due to contamination by the A or B sky background positions. The plotted velocity scale is $V_{\mathrm{lsr}}=-10$ to $+20 \mathrm{~km} \mathrm{~s}^{-1}$, and the intensity scale $T_{\mathrm{R}}^{*}=-25$ to $+25 \mathrm{~K}$.

understand the $\mathrm{CO} J=6 \rightarrow 5$ observed emission. An envelope+disk system centered on EL 29 was resolved by interferometric observations of the ${ }^{13} \mathrm{CO} 1 \rightarrow 0$ transition and has a rest velocity of $v_{\text {LRS }} \sim 5 \mathrm{~km} \mathrm{~s}^{-1}$. It is embedded in a dense ridge which extends NW-SE. The ridge has a velocity similar to the EL 29 envelope+disk system, namely $\sim 5 \mathrm{~km} \mathrm{~s}^{-1}$, and it is probed by the emission of the $\mathrm{HCO}^{+} J=3 \rightarrow 2$ transition (single dish data) and $\mathrm{HCO}^{+} J=1 \rightarrow 0$ (interferometric data). The EL 29 envelope+disk and the ridge are behind two molecular clouds whose rest velocity is $\sim 2.7$ and $\sim 3.8 \mathrm{~km} \mathrm{~s}^{-1}$ respectively. The same situation is visible in the observed CO $J=6 \rightarrow 5$ spectra. In all mapped positions the line is heavily absorbed around $\sim 3.8 \mathrm{~km} \mathrm{~s}^{-1}$ because of the cloud foreground material. Towards EL 29 (at $0^{\prime \prime}, 0^{\prime \prime}$ ) the line is absorbed at $\sim 5 \mathrm{~km} \mathrm{~s}^{-1}$ (Fig. 2), by material belonging to the ridge and/or envelope+disk.

Notably, we detected ${ }^{13} \mathrm{CO} J=6 \rightarrow 5$ emission with $T_{R}^{*}=(10 \pm 1) \mathrm{K}$ towards the central position, whereas we obtained a $3 \sigma$ upper limit of $T_{R}^{*} \leq 2.6 \mathrm{~K}$ at $\left(-14^{\prime \prime},+14^{\prime \prime}\right)$ (Fig. 2), i.e. in a positions still inside the ridge. The on-source ${ }^{13} \mathrm{CO} J=6 \rightarrow 5$ line peaks at $\sim 5 \mathrm{~km} \mathrm{~s}^{-1}$, suggesting that the bulk of the ${ }^{12} \mathrm{CO} \mathrm{J}=6 \rightarrow 5$ emission is due to the envelope+disk and/or ridge associated with EL 29. However, since the on-source ${ }^{13} \mathrm{CO} J=6 \rightarrow 5$ line peak is about half the ${ }^{12} \mathrm{CO}$ line peak and less than 0.15 at $-14^{\prime \prime},+14^{\prime \prime}$, i.e. in

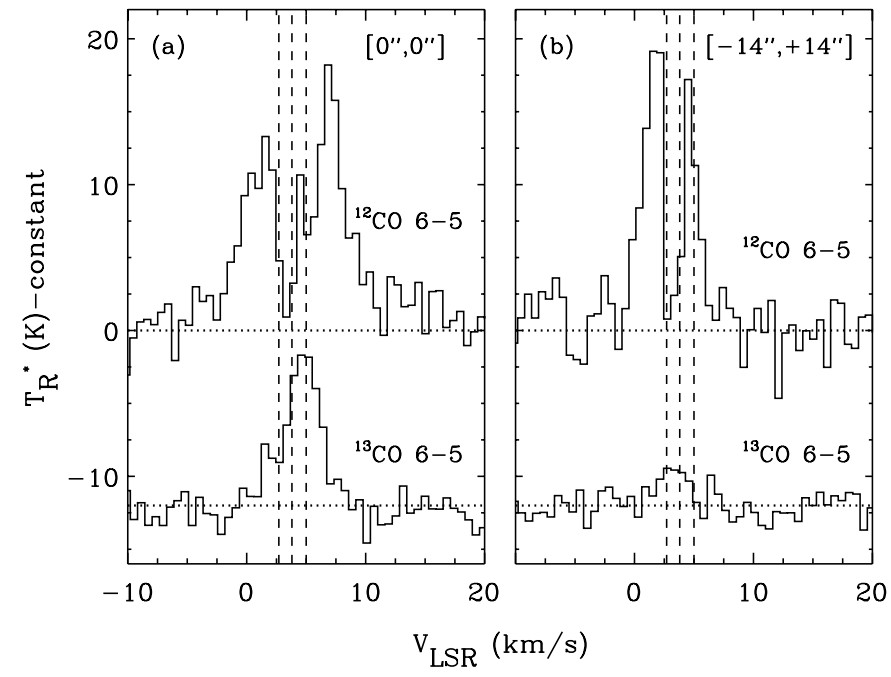

Fig. 2. ${ }^{12} \mathrm{CO}$ (top) and ${ }^{13} \mathrm{CO}$ (bottom) $J=6 \rightarrow 5$ line spectra towards $\left(0^{\prime \prime}, 0^{\prime \prime}\right)$ (left panel) and $\left(-14^{\prime \prime},+14^{\prime \prime}\right)$ (right panel) respectively. Vertical lines mark the rest velocity of the foreground clouds studied in $\mathrm{BHC} 02$.

the ridge, it is likely that most of the absorption (and emission) towards the central position is due to the envelope+disk rather than the ridge. 


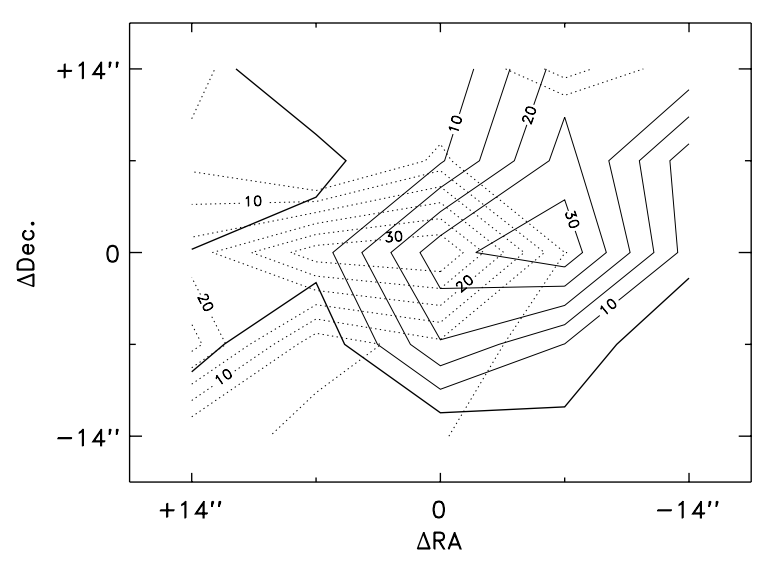

Fig. 3. Contour map of the wing CO $J=6 \rightarrow 5$ emission toward EL 29. The solid lines show the blue shifted emission integrated between -10 and $0 \mathrm{~km} \mathrm{~s}^{-1}$, while the dotted lines show the red shifted emission in the interval +7 to $+17 \mathrm{~km} \mathrm{~s}^{-1}$. Levels of 5, 10, 15, 20, 25, and $30 \mathrm{~K} \mathrm{~km} \mathrm{~s}^{-1}$ are shown.

The ${ }^{12} \mathrm{CO}$ spectra of Fig. 1 present wings at high velocity $\left(\geq 4 \mathrm{~km} \mathrm{~s}^{-1}\right.$ ), which probe the presence of outflowing gas. The map of the wing emission (Fig. 3) shows the characteristic bipolar shape, with the blue lobe on the west side and the red lobe to the east. The outflow traced by our $J=6 \rightarrow 5$ observations is roughly in agreement with that reported by Bontemps et al. (1996) and Sekimoto et al. (1997), obtained in the $2 \rightarrow 1$ transition. However, the morphology of the outflow seems better resolved in the $J=6 \rightarrow 5$ line, probably because of the smaller contamination from the cloud material.

\subsection{FIR line emission}

Figure 4 shows the line spectrum observed towards EL 29, after removal of the continuum (for the continuum analysis look at BHC02). The line spectra towards EL 29 and the off-source position are dominated by the [OI]63 $\mu \mathrm{m}$ and [CII] $158 \mu \mathrm{m}$ lines, which have comparable fluxes at the two (ON and OFF) positions: $6 \times 10^{-12} \mathrm{erg} \mathrm{s}^{-1} \mathrm{~cm}^{-2}$ ([OI]) and $10 \times 10^{-12} \mathrm{erg} \mathrm{s}^{-1} \mathrm{~cm}^{-2}$ ([CII]). The fact that the fluxes are similar at $\mathrm{ON}$ and OFF positions indicates that both lines are (mainly) formed in the "diffuse" material which belongs to the molecular cloud, and very little of the emission, if any, can be attributed to EL 29 itself (see Liseau et al. 1999 for a discussion of the $\mathrm{C}^{+}$and $\mathrm{O}^{\circ}$ emission in the $\rho$ Oph cloud).

In contrast, the ISO observations detected five CO lines, from $J=15$ to $J=19$, only toward EL 29. The line parameters are summarized in Table 1 . The values reported in Table 1 were computed by best fitting a Gaussian whose FWHM is equal to the resolution of the spectrometer and by defining a baseline around each line. The definition of the baseline is also a source of error in the flux estimates (note that the lines are $\sim 1 / 50$ the continuum) and gives around $20 \%$ uncertainty. The statistical errors given in Table 1 are those due to the residuals from the Gaussian fit to the fluxes, and the total error also takes into account uncertainty in the baseline determination. An additional $20 \%$ uncertainty should be considered when considering absolute fluxes. Note that although the total errors reported
Table 1. Parameters of the CO lines observed towards EL 29. First column gives the theoretical line center, second column the observed fluxes with their statistical errors, third column the total error on the flux, including baseline uncertainty (see text), and fourth column the transition. The upper limits are $2 \sigma$ of the noise.

\begin{tabular}{rccc}
\hline $\begin{array}{r}\lambda \\
(\mu \mathrm{m})\end{array}$ & $\begin{array}{c}\text { Flux } \\
10^{-12} \mathrm{erg} \mathrm{s}^{-1} \mathrm{~cm}^{-2}\end{array}$ & $\begin{array}{c}\text { Error } \\
\mathrm{CO}\end{array}$ \\
\hline 130.37 & $\leq 1.0$ & & $20 \rightarrow 19$ \\
137.20 & $0.5 \pm 0.3$ & 0.5 & $19 \rightarrow 18$ \\
144.78 & $1.0 \pm 0.3$ & 0.6 & $18 \rightarrow 17$ \\
153.27 & $1.6 \pm 0.3$ & 0.6 & $17 \rightarrow 16$ \\
162.81 & $1.2 \pm 0.3$ & 0.6 & $16 \rightarrow 15$ \\
173.63 & $1.2 \pm 0.3$ & 0.4 & $15 \rightarrow 14$ \\
185.95 & $\leq 0.8$ & & $14 \rightarrow 13$ \\
\hline
\end{tabular}

in Table 1 are large with respect to the quoted fluxes, they represent only an uncertainty on the flux estimates and not on the line detection, for which one has to consider the statistical errors.

Figure 4 shows the presence of two unidentified lines at $146.8 \mu \mathrm{m}$ and $139.6 \mu \mathrm{m}$ respectively. The only correspondence we found is with $\mathrm{H}_{2} \mathrm{~S}$ lines, but the lack of other detected $\mathrm{H}_{2} \mathrm{~S}$ lines in the spectrum excludes these identifications. Finally, we did not detect any other line, specifically from $\mathrm{H}_{2} \mathrm{O}$, $\mathrm{OH}$ and ${ }^{13} \mathrm{CO}$, at a level of $\sim 0.7 \times 10^{-12} \mathrm{erg} \mathrm{s}^{-1} \mathrm{~cm}^{-2}(2 \sigma \mathrm{rms})$. An apparent peak close to the $\mathrm{CO}$ line at $163 \mu \mathrm{m}$ is far too red to be associated with the $\mathrm{OH}$ line at $163.4 \mu \mathrm{m}$. There may be some $\mathrm{NH}_{3}$ emission responsible for the feature visible at $170 \mu \mathrm{m}$, but given the uncertainty in the existence of such a feature we do not explore further this possibility. $\mathrm{No}_{2}$ lines were detected in the SWS spectrum and the obtained $2 \sigma$ upper limits are 1 and $2 \times 10^{-12} \mathrm{erg} \mathrm{s}^{-1} \mathrm{~cm}^{-2}$ for the $\mathrm{S}(1)$ and $\mathrm{S}(2)$ lines, and $6 \times 10^{-13} \mathrm{erg} \mathrm{s}^{-1} \mathrm{~cm}^{-2}$ for the other lines.

\section{LVG modeling of the $\mathrm{CO}$ emission}

As discussed in Sect. 3, the CO $J=6 \rightarrow 5$ emission is due to two components: the dense envelope+disk system, probed by the ${ }^{13} \mathrm{CO}$ emission, whose linewidth is $\sim 3.6 \mathrm{~km} \mathrm{~s}^{-1}$ (Fig. 2) and the outflow, probed by the ${ }^{12} \mathrm{CO}$ wing emission, whose linewidth is $\sim 10 \mathrm{~km} \mathrm{~s}^{-1}$ (Fig. 3). Both components are in principle within the $80^{\prime \prime}$ LWS beam and therefore the observed CO $J=15$ to $J=20$ emission may be due to either or both of them, or, finally, none of them. Unfortunately, the spectral resolution of the ISO observations is not enough to disentangle the two contributions so that the origin of the CO $J=15$ to $J=20$ emission is unclear based only on the observational facts. We will therefore try to use theoretical arguments to shed light on the origin of the $J \geq 15$ emission.

To interpret the observed $\mathrm{CO}$ spectrum we used an LVG model, applied to a slab geometry, where the escape probability depends on the line opacity through the relation (Scoville \& Solomon 1974): $\beta=\frac{1-\exp [-3 \tau]}{3 \tau}$. Our model considers the first 50 rotational levels, with the collisional excitation rates taken from McKee et al. (1982). More details on 


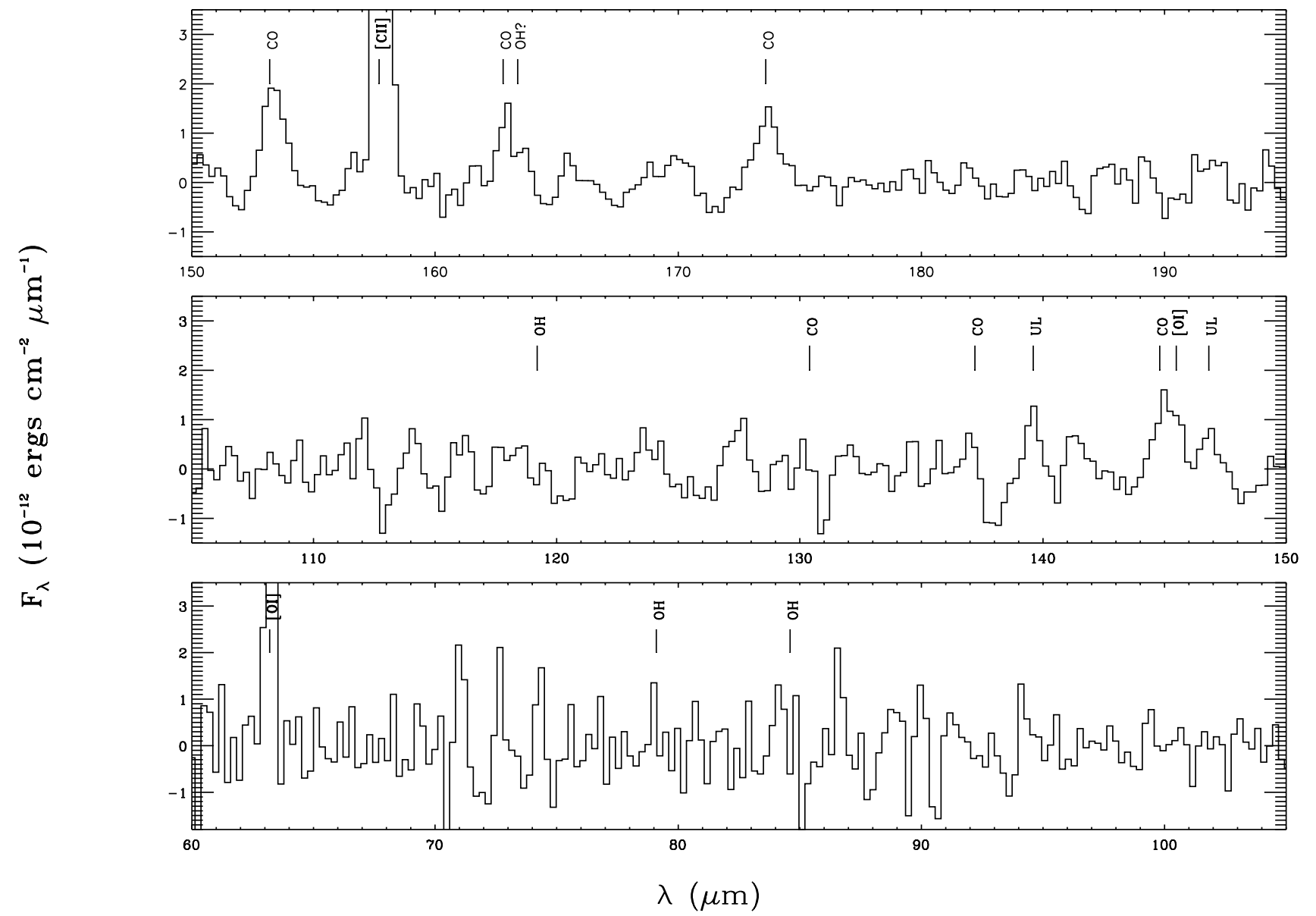

Fig. 4. The line spectrum of EL 29 after removal of the continuum. The expected brightest lines are marked from $\mathrm{O}^{0}, \mathrm{C}^{+}, \mathrm{CO}, \mathrm{H}_{2} \mathrm{O}$ and $\mathrm{OH}$ species. Two lines clearly detected at $146.8 \mu \mathrm{m}$ and $139.6 \mu \mathrm{m}$ are unidentified and marked as UL in the figure.

the code used and formalism can be found in Ceccarelli et al. (2002). In the present computations we neglect the dust radiation pumping of the $\mathrm{CO}$ levels. The computed $\mathrm{CO}$ line emission depends on five parameters: the density and the temperature of the gas, the linewidth, the angular extent of the emitting region and the CO column density. We ran several models spanning a large parameter space: density between $10^{5}$ and $10^{8} \mathrm{~cm}^{-3}$, temperature between $50 \mathrm{~K}$ and $800 \mathrm{~K}, \mathrm{~N}(\mathrm{CO})$ between $10^{16}$ and $10^{19} \mathrm{~cm}^{-2}$.

When the lines are optically thin (i.e. $N(\mathrm{CO}) \leq 10^{18} \mathrm{~cm}^{-3}$ ) the line ratios do not depend on the $\mathrm{CO}$ column density and can be used to constrain approximatively the gas temperature and density, whereas when the lines are moderately optically thick, the line ratios also depend on the CO column density. Even assuming that the $J \geq 15$ lines are optically thin the observed line ratios yield only loose constraints: the gas temperature is higher than $\sim 250 \mathrm{~K}$ and the density is higher than $\sim 3 \times 10^{5} \mathrm{~cm}^{-3}$. Allowing the lines to be optically thick, which in principle is possible, does not improve the situation much. Practically, modeling of the $J \geq 15$ observed emission does not help to understand its origin.

We therefore try to model the $\mathrm{CO} J=6 \rightarrow 5$ emission and to see whether it can help to understand the origin of the $J=15$ to $J=20$ emission too. As discussed in Sect. 3, the $\mathrm{CO} J=6 \rightarrow 5$ emission observed in the central position originates mainly in the envelope+disk. These observations, and in particular the ${ }^{13} \mathrm{CO}$ ones, turn out to be the key to understand where the FIR CO lines originate. To interpret the observed $J=6$ to $J=20$ lines, we use the LVG model described above. Although a LVG model is by definition a rough description of the emission associated with the envelope+disk, whose temperature and density is far from constant, we think it is worth having a first approximation of the average density and temperature of the gas responsible for the $\mathrm{CO}$ emission. We assume then that the emission is generated by a gas whose linewidth is $3.6 \mathrm{~km} \mathrm{~s}^{-1}$ (Fig. 2) and whose extent is smaller than the beam of the $\mathrm{CO} J=6 \rightarrow 5$ observations, i.e. $12^{\prime \prime} \times 12^{\prime \prime}$.

The fact that the ${ }^{13} \mathrm{CO} J=6 \rightarrow 5$ line intensity is half the ${ }^{12} \mathrm{CO}$ intensity puts a stringent constraint on the line opacity and consequently on the $\mathrm{CO}$ column density, the emitting sizes and gas temperature. Taking the isotope abundance ${ }^{12} \mathrm{CO} /{ }^{13} \mathrm{CO}=80$ (Boogert et al. 2000), the observed line ratio implies $\tau \sim 15$ and a $\mathrm{CO}$ column density between 3 and $7 \times 10^{18} \mathrm{~cm}^{-2}$. The exact column density value depends on the gas temperature: if $N(\mathrm{CO})=3 \times 10^{18} \mathrm{~cm}^{-2}$ then the gas temperature is $T_{\text {gas }}=90 \mathrm{~K}$ and the size is $\Delta \theta=5^{\prime \prime}$; the upper value $N(\mathrm{CO})=7 \times 10^{18} \mathrm{~cm}^{-2}$ implies $T_{\text {gas }}=200 \mathrm{~K}$ and $\Delta \theta=3^{\prime \prime}$. In the following we consider the median values: $N(\mathrm{CO})=5 \times 10^{18} \mathrm{~cm}^{-2}, T_{\text {gas }}=170 \mathrm{~K}$ and $\Delta \theta=4^{\prime \prime}(=550 \mathrm{AU}$ for a distance of $160 \mathrm{pc}$ ). Using the parameters derived from 
Table 2. Warm gas: parameters derived from the LVG analysis of the CO submm and FIR line emission towards EL 29.

\begin{tabular}{lr}
\hline \hline Parameter & Value \\
\hline Temperature & $170-250 \mathrm{~K}$ \\
Density & $\geq 10^{6} \mathrm{~cm}^{-3}$ \\
Size & $4^{\prime \prime}=550 \mathrm{AU}$ \\
$N(\mathrm{CO})$ & $5 \times 10^{18} \mathrm{~cm}^{-2}$ \\
$\mathrm{CO} / \mathrm{H}$ & $1-3 \times 10^{-4}$ \\
Mass & $8-24 \times 10^{-4} M_{\odot}$ \\
\hline
\end{tabular}

the $J=6 \rightarrow 5$ observations, we computed the $J=15$ to $J=20$ CO spectrum for a gas density from $10^{6}$ to $10^{8} \mathrm{~cm}^{-3}$ (Fig. 5). Although the $\mathrm{CO} J=6 \rightarrow 5$ data do not allow us to constrain the density, we can reasonably assume that it is higher than at least $10^{6} \mathrm{~cm}^{-3}$, as the (more extended) ridge in which EL 29 is embedded has itself a density of $\sim 5 \times 10^{5} \mathrm{~cm}^{-3}$ (BHC02). Actually, the model by Chiang \& Goldreich (1997; hereinafter CG97) which fits the EL 29 continuum spectrum predicts a midplane disk density of $\sim 10^{9} \mathrm{~cm}^{-3}$ at $\sim 250 \mathrm{AU}$ (BHC02), the size derived by the $\mathrm{CO} J=6 \rightarrow 5$. Figure 5 shows then that most, if not all of the $J \geq 15$ emission is also due to this envelope+disk component. Gradients in the density and/or temperature would certainly account for the minor differences between the observed and predicted FIR fluxes.

The upper limits on the $\mathrm{H}_{2}$ line fluxes put some stringent limit on the $\mathrm{CO}$ abundance. Assuming LTE and optically thin lines, as is likely for the $\mathrm{H}_{2}$ rotational lines under consideration, the upper limits on the observed fluxes translate into an upper limit to the $\mathrm{H}_{2}$ column density, and hence a lower limit to the $\mathrm{CO}$ abundance $x(\mathrm{CO})=N(\mathrm{CO}) /\left(2 \times N\left(\mathrm{H}_{2}\right)\right)$. Taking into account the high extinction toward EL 29 ( 27 mag; Boogert et al. 2000, 2002) and correcting for it assuming the $A_{\lambda} / A_{v}$ ratio by Lutz et al. (1996), gives $\mathrm{CO} / H \geq 1 \times 10^{-4}$. In practice, as expected based on the temperature, $\mathrm{CO}$ is not depleted in the warm region. It is worth noting that $\mathrm{CO}$ is not photo-dissociated either, probably because of the shielding from the remaining envelope. Finally, the computed ${ }^{13} \mathrm{CO}$ FIR line fluxes $\left(\leq 5 \times 10^{-14} \mathrm{erg} \mathrm{s}^{-1} \mathrm{~cm}^{-2}\right)$ are below the LWS detection limit and therefore consistent with their non-detection, despite the fact that ${ }^{12} \mathrm{CO}$ lines are moderately optically thick. The non-detection of water lines implies a $\mathrm{H}_{2} \mathrm{O}$ column density lower than $\sim 10^{16} \mathrm{~cm}^{-2}$, giving a water abundance $\leq 5 \times 10^{-7}$ (assuming the largest possible $\mathrm{CO}$ abundance, i.e. $\mathrm{CO} / \mathrm{H}=$ $3 \times 10^{-4}$; Lacy et al. 1994) in the gas emitting the warm CO.

In summary, the $\mathrm{CO} J=6, J=15$ to $J=20 \mathrm{ob}-$ served emission may originate in a component located at about $250 \mathrm{AU}$ from the center and whose mean density is $\geq 10^{6} \mathrm{~cm}^{-3}$ and temperature $\sim 170 \mathrm{~K}$ (Table 2 ). The mass of this warm gas amounts to $\sim 8 \times 10^{-4} M_{\odot}$ (assuming $\mathrm{CO} / \mathrm{H}=1 \times 10^{-4}$; a larger $\mathrm{CO}$ abundance would give a linearly lower warm gas mass). It is worth emphasizing that this result stems mostly from the interpretation of the $\mathrm{CO} J=6 \rightarrow 5$ observations $\left({ }^{12} \mathrm{C}\right.$ and ${ }^{13} \mathrm{C}$ observations), without further adjustments other than the density to explain the $J=15$ to $J=19$ observed emission too.

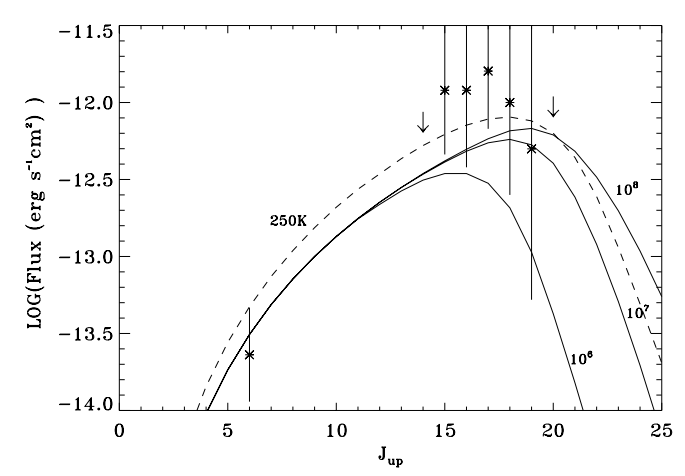

Fig. 5. Observed and modeled CO spectrum towards EL 29. Fluxes are in erg $\mathrm{s}^{-1} \mathrm{~cm}^{-2}$. Solid lines represent a model with $N(\mathrm{CO})=$ $5 \times 10^{18} \mathrm{~cm}^{-2}$, emitting sizes equal to $4^{\prime \prime}$, the gas temperature equal to $170 \mathrm{~K}$, and density from $10^{6}$ to $10^{8} \mathrm{~cm}^{-3}$, as marked in the plot. The dashed line shows a case with density equal to $10^{6} \mathrm{~cm}^{-3}$ and a temperature equal to $250 \mathrm{~K}$. Note that the error bars do not include the $20 \%$ absolute calibration uncertainty in the $J \geq 15$ lines with respect to the $J=6$ line.

\section{The disk model}

The CO $J=6 \rightarrow 5$ linewidth of $3.6 \mathrm{~km} \mathrm{~s}^{-1}$ is consistent with outflowing gas, but it can also be explained by gas infalling and/or orbiting towards a $\sim 1 M_{\odot}$ central object. We already mentioned that the outflow/shock thesis has difficulty explaining why the water abundance is so low, less than $5 \times 10^{-7}$ in the specific case of El 29. In the following we explore as far as possible the hypothesis that the observed $\mathrm{CO}$ emission originates in the envelope+disk of EL 29. In an extensive study, BHC02 modeled the 2 to $1300 \mu \mathrm{m}$ continuum spectrum (SED) of EL 29 and found that it can be reproduced by a relic envelope that surrounds a flaring disk whose mass is about $0.012 M_{\odot}$. The same model predicts a contribution from the envelope to the ${ }^{12} \mathrm{CO} J=6 \rightarrow 5$ emission equal to $T_{\mathrm{R}}^{*}=18 \mathrm{~K}$, i.e. a substantial fraction compared to the observed signal $\left(T_{\mathrm{R}}^{*}=\right.$ $20 \mathrm{~K})$. However, the ${ }^{13} \mathrm{CO} J=6 \rightarrow 5$ emission from the envelope would result in $T_{\mathrm{R}}^{*}=1.5 \mathrm{~K}$, compared to the observed $10 \mathrm{~K}$. Similar results are obtained using the model of the envelope emission by Ceccarelli et al. (1996). Both models predict by far too low CO FIR line emission with respect to what is observed. We therefore conclude that the envelope does not contribute substantially to the observed CO FIR and ${ }^{13} \mathrm{CO} J=6 \rightarrow 5$ emission (although the envelope dominates the ${ }^{12} \mathrm{CO} J=6 \rightarrow 5$ emission), unless a key ingredient is missing in the two models mentioned. A possibility is the existence of a "super-heated" envelope layer illuminated by the UV and/or X-ray emitted at the center, which neither the $\mathrm{BHCO} 2$ or Ceccarelli et al. models include.

We hence explore the possibility that the disk is the main cause of the observed CO submm/FIR emission. In BHC02 we used the disk model by CG97 that predicts the existence of a disk "super-heated surface layer". To reproduce the SED of EL 29 we used the CG97 temperature profile multiplied by a factor of 2.5 to account for the EL 29 luminosity (BHC02). This corresponds to a dust temperature of the super-heated layer at $250 \mathrm{AU}$ of about $160 \mathrm{~K}$, in remarkable agreement with the gas temperature that we find from the $\mathrm{CO}$ submm/FIR line $\mathrm{LVG}$ 
analysis. In addition, we found that the mass of the superheated layer is $\sim 4-20 \times 10^{-4} M_{\odot}$, in remarkable agreement with that derived from the LVG analysis of the CO submm/FIR line data $\left(\sim 8-24 \times 10^{-4} M_{\odot}\right)$. Even considering the various approximations in the analysis of both the continuum and line data, the substantial agreement of the derived disk parameters seems to be extremely encouraging and supportive of the thesis that the FIR CO emission originates in the super-heated layer of the EL 29 disk. We therefore computed the CO line emission from the flaring disk model which also fits the SED, hereinafter referred to as the $\mathrm{BHC} 02$ model. As expected, the $\mathrm{CO}$ column density predicted by the BHCO2 model (about $5 \times 10^{18} \mathrm{~cm}^{-2}$ in a 4 " region) agrees well with the required column density. In addition, we have computed the $\mathrm{CO}$ line emission and find that the predicted $J=6 \rightarrow 5,{ }^{12} \mathrm{CO}$ and ${ }^{13} \mathrm{CO}$ fluxes are comparable to the observed values.

On the contrary, the predicted FIR CO line fluxes are a factor 10 lower than the observed values. However, we think that this discrepancy is easily explained. Indeed, in the flaring disk used for the SED analysis we assumed the minimum density for the super-heated layer provided by CG97. As a consequence, the density in the BHCO2 model is relatively low, being only $1 / 3$ of the material in the super-heated layer with a density above $10^{6} \mathrm{~cm}^{-3}$ and only $1 / 10^{4}$ above $10^{7} \mathrm{~cm}^{-3}$. This explains why the FIR CO lines are not excited in this model. However, as CG97 themselves caution, the density in the super-heated layer can be some orders of magnitude larger that the minimum we used in the $\mathrm{BHCO} 2$ model. The maximum density would be that of the disk midplane if the dust had fully settled, e.g. $10^{9} \mathrm{~cm}^{-3}$ at $250 \mathrm{pc}$. Hence the density in the super-heated layer can be anything between $10^{6} \mathrm{~cm}^{-3}$ and $10^{9} \mathrm{~cm}^{-3}$. Since the SED analysis is not sensitive to the density but just to the column density of the dust, the BHCO2 model could not constrain it. The present line observations may suggest a rather high density and therefore that the dust settling has already progressed in EL 29. Alternatively, the LVG modeling (Fig. 5) shows that lower densities are still possible but would require a somewhat larger temperature, $\sim 250 \mathrm{~K}$, to account for the $J=6$ to $J=20$ observed emission together. From a theoretical point of view it would not be impossible that the gas and dust are thermally decoupled, with the gas warmer than the dust. From an observational point of view this seems rather the case (see next paragraph). Given all these uncertainties it is difficult to push further the $\mathrm{BHC} 02$ model/observations comparison.

Unfortunately, both the continuum and line data are not accurate enough to explore in more detail the scenario of whether the gas is decoupled from the dust and how much (a short discussion on this possibility is reported in the next section). Recently D'Alessio et al. $(1999,2001)$ modeled in a selfconsistent way the vertical structure of flaring disks (Calvet et al. 1991; CG97) and found that their models predict too (geometrically) thick disks with respect to the observed T Tauri stars. They suggested that dust settling and/or dust coagulation could possible resolve this apparent contradiction between predictions and observations, even though the opacity is set by the small grains which settle with more difficulty. The present observations seem to support the thesis of dust settling in EL 29. However, it is clear is that the density, temperature and

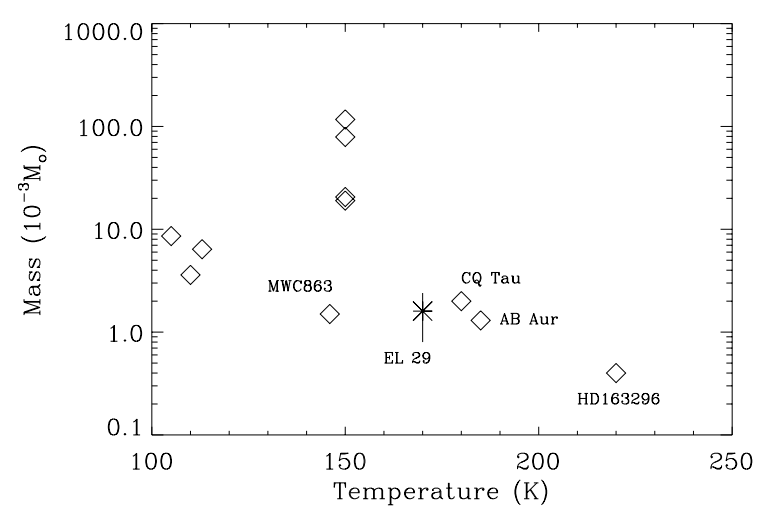

Fig. 6. Masses and temperatures measured by the $\mathrm{H}_{2}$ observations of the Thi et al. (2001) sample (diamonds) and by our observations in EL 29 (asterisk). Some Herbig AeBe stars of the Thi et al. (2001) sample are marked for reference.

chemical structure of the disk are crucial parameters for a fully consistent modeling of line emission, but this is beyond the scope of this article, also because of the too large observational uncertainties. The main point we want to stress is that observationally the CO FIR emission indicates a reservoir of relatively dense and warm gas of mass about that of the dusty super-heated layer derived by the SED analysis. We therefore think that the hypothesis that the CO submm/FIR lines originate in the disk is very reasonable.

\section{Discussion}

Indeed the disk hypothesis is not surprising in the light of the recent analysis of the $\mathrm{H}_{2}$ and $\mathrm{CO} J=6 \rightarrow 5$ emission in T Tauri and Herbig AeBe stars by Thi et al. (2001). They found that the surveyed stars (about a dozen) have relatively large amounts, $\sim 10^{-2}-10^{-3} M_{\odot}$, of warm gas, whose temperature varies between $100 \mathrm{~K}$ and $220 \mathrm{~K}$. The T Tauri star sample have gas at $\sim 100 \mathrm{~K}$, whereas Herbig AeBe stars have higher temperatures, $\geq 150 \mathrm{~K}$. Figure 6 plots the masses and temperatures measured by the $\mathrm{H}_{2}$ observations of the Thi et al. sample together with the values that we derived for EL 29. This figure shows that EL 29 has a "normal" disk, when compared to the Herbig AeBe stars of the sample. Since EL 29 is a probable precursor of Herbig AeBe stars (e.g. BHC02), in this respect and on a purely observational basis, EL 29 does not seem to possess a particularly peculiar disk, a fact that strengthens further the thesis that FIR CO emission originates in its disk. More specifically, AB Aur (in the sample of Thi et al. 2001) shares many similarities with EL 29. AB Aur and EL 29 have a bolometric luminosity of $\sim 50$ and $\sim 40 L_{\odot}$ respectively, similar flat SEDs, and relatively face-on disks. The AB Aur $\mathrm{H}_{2}$ observations yield a gas temperature of $(185 \pm 15) \mathrm{K}$, against the $170-250 \mathrm{~K}$ we find for EL 29, and a warm gas mass of $(1.3 \pm 0.7) \times 10^{-3} M_{\odot}$, against $8-20 \times 10^{-4} M_{\odot}$ in EL 29 . As a matter of fact, the ${ }^{12} \mathrm{CO} J=6 \rightarrow 5$ intensity is very similar to that of EL $29\left(50\right.$ against $\left.100 \mathrm{~K} \mathrm{~km} \mathrm{~s}^{-1}\right)$ and the $S(1)$ $\mathrm{H}_{2}$ line flux is $3 \times 10^{-13} \mathrm{erg} \mathrm{s}^{-1} \mathrm{~cm}^{-2}$ in $\mathrm{AB}$ Aur, consistent with the upper limit for EL 29 of $10 \times 10^{-13} \mathrm{erg} \mathrm{s}^{-1} \mathrm{~cm}^{-2}$. The ${ }^{12} \mathrm{CO} J=6 \rightarrow 5$ linewidth is $2.1 \mathrm{~km} \mathrm{~s}^{-1}$ in $\mathrm{AB}$ Aur against 


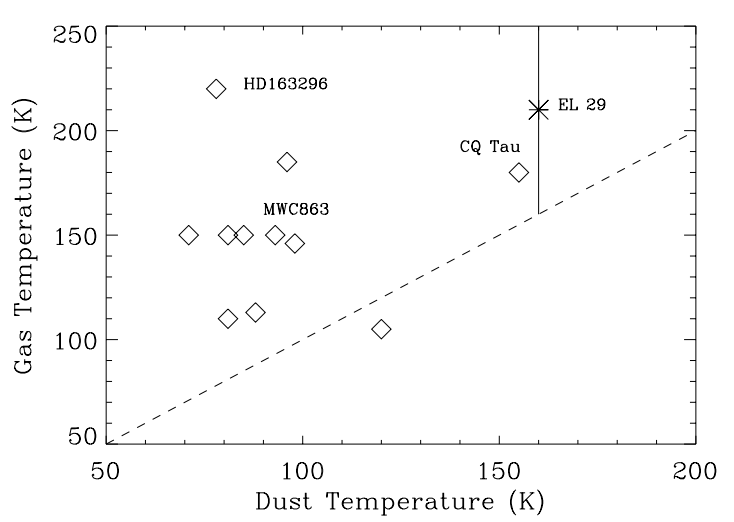

Fig. 7. Gas and dust temperatures measured by the $\mathrm{H}_{2}$ observations of the Thi et al. (2001) sample (diamonds) and by our observations in EL 29 (asterisk). Some Herbig AeBe stars of the Thi et al. (2001) sample are marked for reference. The dashed line marks the gas temperature equal to the dust temperature.

the $3.6 \mathrm{~km} \mathrm{~s}^{-1}$ in EL 29, perhaps because of a slightly different inclination angle. As already remarked, it is likely that the ${ }^{12} \mathrm{CO} J=6 \rightarrow 5$ in EL 29 is strongly "contaminated" by the envelope and some of the ridge in which EL 29 is embedded, more than $\mathrm{AB}$ Aur is, and that would explain the stronger and relatively extended ${ }^{12} \mathrm{CO} J=6 \rightarrow 5$ emission in EL 29 . Class I (embedded) sources are indeed known to possess envelopes that emit copiously in the CO $J=6 \rightarrow 5$ transition (Hogerheijde et al. 1998). For example, L1489 IRS has a ${ }^{12} \mathrm{CO} J=6 \rightarrow 5$ intensity very similar to that in EL 29.

We mentioned above the possibility that the gas temperature of the EL 29 super-heated layer is somewhat larger than the dust temperature. There are reasons to think that gas and dust are thermally decoupled in the disk super-heated layer. First and foremost, from an observational point of view this is exactly what has been observed in the disks of T Tauri and Herbig AeBe stars by Thi et al. (2001). They found that the gas temperature derived by the observed $\mathrm{H}_{2}$ lines is systematically $20 \mathrm{~K}$ or more larger than the dust temperature derived by the continuum at the frequency of the $\mathrm{H}_{2}$ lines. In this respect, once again, EL 29 does not constitute a peculiar case, as shown in Fig. 7. As Thi et al. $(1999,2001)$ discuss in their articles, the gas may be warmer because of UV photon heating. Here we wish to address the possibility that X-rays from the central source can also play a role in this extra heating (e.g. Glassgold et al. 1997), as Pre-Main Sequence stars are known to be prodigious X-rays emitters (Feigelson \& Montemerle 1999). In particular EL 29 emits about $5 \times 10^{-4} L_{\odot}$ in the X-ray and it is known to be a X-ray flaring source too (Imanishi et al. 2001). We postpone, however, a full discussion of the heating mechanisms to a forthcoming paper (Ceccarelli et al. in prep.). Whether and how much the gas and dust are decoupled in EL 29 is difficult to establish on the basis of the available observations. Further observations should be able to settle the question.

Finally, we wish to comment the presumed problem of water underabundance (see Introduction). Our LWS observations show that the water abundance is less than $5 \times 10^{-7}$ in the superheated layer disk of EL 29. This value does not present any "problem" and it is consistent with theoretical expectations of chemical models (e.g. Lee et al. 1996). Indeed the water abundance in the gas around low mass protostellar envelopes is few times $10^{-7}$ (e.g. Ceccarelli et al. 2000; Maret et al. 2002) and in the molecular clouds is even lower (Caux et al. 1999; Snell et al. 2000). Since the gas temperature does not exceed the fatal threshold of $\sim 250 \mathrm{~K}$, needed to open the route to water formation by endothermic reactions (e.g. Wagner \& Graff 1987), there is no reason to expect an enhanced water abundance in the super-heated layer, even if a large fraction of gaseous water is injected from the evaporated grain mantles. In fact the superheated layer is by definition exposed to the UV photons from the central source and most of water would hence be photodissociated (also in the region where $\mathrm{CO}$ is re-formed, because of the self-shielding). The upper limit on the water abundance that we find is, in this respect, in good agreement with the value found in the PDR of NGC $133\left(\sim 10^{-7}\right.$ by Bergin et al. 2002). Incidently, this implies that $\mathrm{H}_{2} \mathrm{O}$ line emission is not the main gas cooling mechanism as CG97 assumed in their study of the disk characteristics. The EL 29 LWS spectrum shows that CO is indeed the main coolant of the gas in the disk super-heated layer. Since CO is a relatively low efficiency gas cooler, the gas temperature can stay higher than they presumed in their article.

\section{Conclusions and perspectives}

We presented observations of the $\mathrm{CO}$ emission from $J=6$ to $J=19$ and $\mathrm{H}_{2}$ emission, observed towards EL 29 with different instruments, ISO and JCMT. The simultaneous analysis of all these data suggests that the submm/FIR CO emission, $J=6$ and between $J=15$ and $J=19$ is emitted by a $\sim 4$ " (i.e. $\sim 250 \mathrm{AU}$ from the center) region of gas, whose temperature is between $170 \mathrm{~K}$ and $250 \mathrm{~K}$, whose density is larger than $\sim 10^{6} \mathrm{~cm}^{-3}$ and whose $\mathrm{CO}$ column density is $5 \times 10^{18} \mathrm{~cm}^{-2}$. The mass of the warm gas is $8-24 \times 10^{-4} M_{\odot}$ and its CO abundance is $\geq 1 \times 10^{-4}$, implying no substantial $\mathrm{CO}$ depletion and/or photodissociation. We propose that this warm gas resides in a super-heated surface disk layer. We applied the disk model previously developed to reproduce the SED of EL 29 (BHC02) and could also reproduce the observed submm/FIR CO lines, assuming a relatively large density in the heated layer $\left(\geq 10^{6} \mathrm{~cm}^{-3}\right)$. This density is expected when the dust has started to settle to the midplane. Alternatively, dust and gas may be largely thermally decoupled, with the gas temperature about $250 \mathrm{~K}$.

The derived temperature and mass are similar to the values found in the disks of other Herbig AeBe stars (Thi et al. 2001), supporting the case that EL 29 is rather a precursor or very embedded Herbig AeBe star. Finally, the lack of water emission is fully consistent with the interpretation of the FIR CO emission originating in the disk of EL 29, and does not necessitate any mechanism to lower the water abundance.

The question arises whether the FIR CO emission observed in several other young protostars is also probing their disks, rather than the so-far claimed shocks (e.g. Giannini et al. 2001). Although a full discussion is out of the scope of this paper and we postpone it to a forthcoming paper, we wish to remark that this theory - FIR CO emitted in the super-heated surface layer of disks - would naturally explain the lack of water emission 
in Class I protostars, as water abundance is expected to be low in the disks. On the contrary, the water emission observed in Class 0 sources is naturally explained in terms of thermal emission from the massive envelopes that surround these sources (Ceccarelli et al. 1996; Ceccarelli et al. 2000; Maret et al. 2002), and has hence a different origin than the $\mathrm{CO}$ emission.

Acknowledgements. We wish to thank P. André, S. Molinari, H. Smith and G. White for their contribution in the first stage of this project. $\mathrm{CC}$ thanks Wing-Fai Thi for making her aware of his thesis work. We finally thank the referee, G. J. van Zadelhoff, for carefully reading the manuscript.

\section{References}

Bachiller, R., Cernicharo, J., Martin-Pintado, J., Tafalla, M., \& Lazareff, B. 1990, A\&A, 231, 174

Bergin, E. A., Neufeld, D. A., \& Melnick, G. J. 1999, ApJ, 510, L145

Bergin, E. A., Kaufman, M. J., Melnick, G. J., Snell, R. L., \& Howe, J. E. 2002, ApJ, in press

Bontemps, S., André, P., Terebey, S., \& Cabrit, S. 1996, A\&A, 311, 858

Boogert, A. C. A., Tielens, A. G. G. M., Ceccarelli, C., et al. 2000, A\&A, 360, 683

Boogert, A. C. A., Hogerheijdeet, M. R., Ceccarelli, C., et al. 2002, ApJ, 570, 708 (BHC02)

Calvet, N., Patino, A., Magris, G., \& D'Alessio, P. 1991, ApJ, 380, 617

Caselli, P., Hartquist, T. W., \& Havnes, O. 1997, A\&A, 322, 296

Caux, E., Ceccarelli, C., Castets, A., et al. 1999, A\&A, 347, L1

Castets, A., Ceccarelli, C., Loinard, L., Caux, E., \& Lefloch, B. 2001, A\&A, 375, 40

Ceccarelli, C., Hollenbach, D. J., \& Tielens, A. G. G. M. 1996, ApJ, 471,400

Ceccarelli, C. 2000, ISO beyonds the Peaks, ESA SP-456, 141

Ceccarelli, C., Caux, E., Loinard, L., et al. 1999, A\&A, 342, L21

Ceccarelli, C., Castets, A., Caux, E., et al. 2000, A\&A, 355, 1129

Ceccarelli, C., Baluteau, J-P., Walmsley, M., et al. 2002, A\&A, 383, 603

Cernicharo, J., Pardo, J. R., Gonzlez-Alfonso, E., et al. 1999, ApJ, 520, L131

Chen, H., Myers, P. C., Ladd, E. F., \& Wood, D. O. S. 1995, ApJ, 445, 377
Chiang, E. I., \& Goldreich, P. 1997, ApJ, 490, 368

Clegg, P. E. C., Ade, P. A. R., Armand, C., et al. 1996, A\&A, 315, L38

Curiel, S., Torrelles, J. M., Rodriguez, L. F., Gomez, J. F., \& Anglada, G. 1999, ApJ, 527, 310

D’Alessio, P. Calvet, N., Hartmann, L., Lizano, S., \& Cantó, J. 1999, ApJ, 527, 893

D’Alessio, P., Calvet, N., \& Hartmann, L. 2001, ApJ, 553, 321

de Graauw, T., Haser, L. N., Beintema, D. A., et al. 1996, A\&A, 315, L49

Feigelson, E. D., \& Montemerle, T. 1999, ARA\&A, 37, 363

Garay, G., Mardones, D., Rodriguez, L-P., Caselli, P., \& Bourke, T. 2002, ApJ, 567, 980

Glassgold, A. E., Najita, J., \& Igea, J. 1997, ApJ, 480, 344

Giannini, T., Nisini, B., \& Lorenzetti, D. 2001, ApJ, 555, 40

Harwit, M., Neufeld, D. A., Melnick, G. J., \& Kaufman, M. J. 1998, ApJ, 497, L105

Hogerheijde, M. R., van Dishoeck, E., Blake, G., \& van Langevelde, H. J. 1998, ApJ, 502, 315

Hollenbach, D. J., \& McKee, C. 1989, ApJ, 342, 306

Imanishi, K., Koyama, K., \& Tsuboi, Y. 2001, ApJ, 557, 747

Kaufamn, M. J., \& Neufeld, D. A. 1996, ApJ, 456, 611

Lacy, J. H., Knacke, R., Geballe, T. R., \& Tokunaga, A. T. 1994, ApJ, 428, L69

Lee, H.-H., Bettens, R. P. A., \& Herbst, E. 1996, A\&AS, 119, 111

Liseau, R., Ceccarelli, C, Larsson, B., Nisini, B., et al. 1996, A\&A, 315, L181

Liseau, R., White, G. J., Larsson, B., Sidher, S., et al. 1999, A\&A, 344, 342

Maret, S., Ceccarelli, C., Caux, E., Tielens, A. G. G. M., \& Castets, A. 2002, A\&A, 395, 573

McKee, C. F., Storey, J. W. V., Watson, D. M., \& Green, S. 1982, ApJ, 259,647

Nisini, B., Benedettini, M., Giannini, T., et al. 2000, A\&A, 360, 297

Schilke, P., Walmsley, C. M., Pineau des Forets, G., \& Flower, D. R. 1997, A\&A, 321, 293

Scoville, N. Z., \& Solomon, P. M. 1974, ApJ, 187, L67

Sekimoto, Y., Tatematsu, K., Umemoto, T., et al. 1997, ApJ, 489, L63

Snell, R. L., Howe, J. E., Ashby, M. L. N., et al. 2000, ApJ, 539, L101

Thi, W. F., van Dishoeck, E. F., Blake, G. A., van Zadelhoff, G. J., \& Hogerheijde, M. R. 1999, ApJ, 521, L63

Thi, W. F., van Dishoeck, E. F., Blake, G. A., et al. 2001, ApJ, 561, 1074

Wagner, A. F., \& Graff, M. M 1987, ApJ, 317, 423

Wakelam, V., Castets, A., Ceccarelli, C., et al. 2002, A\&A, submitted

Wilking, B. A., Lada, C. J., \& Young, E. T. 1989, ApJ, 340, 823 